\title{
Aconselhamento farmacêutico na alta hospitalar: adaptação transcultural de um questionário de satisfação
}

\author{
Pharmaceutical counseling at discharge: cross-cultural adaptation of a satisfaction questionnaire \\ Entrevista farmacéutica al alta: adaptación transcultural de un cuestionario de satisfacción
}

\section{Resumo}

A avalição da satisfação de pacientes com serviços de saúde, por meio de instrumentos validados, permite identificar a necessidade de melhorias e conhecer o impacto das intervenções implementadas. O objetivo deste estudo foi realizar a adaptação transcultural para o contexto brasileiro de um questionário de satisfação do paciente com um serviço abrangente de aconselhamento farmacêutico na alta hospitalar. O questionário é composto por nove itens que avaliam a satisfação com o serviço, além do interesse e compreensão do paciente com as orientações recebidas na alta. $\mathrm{O}$ processo envolveu a tradução, retrotradução e adaptação dos itens e escalas de resposta. A avaliação das equivalências semântica, conceitual, cultural e idiomática entre o questionário original e adaptado foi realizada por um comitê de especialistas, sendo o grau de concordância entre eles medido pelo Coeficiente de Validade de Conteúdo (CVC). A versão final do questionário foi aplicada, por autoadministração, a uma amostra da população-alvo composta por 21 indivíduos, no momento da alta hospitalar. Embora o instrumento adaptado tenha apresentado equivalência satisfatória com o original (CVC>0,70), a população-alvo teve dificuldades consideráveis em compreendê-lo, o que sugere a necessidade de aperfeiçoamento dos itens, por meio de procedimentos teóricos, para que o questionário possa ser submetido a avaliação de suas propriedades psicométricas e, assim, ser utilizado no Brasil.

Palavras-chave: Reconciliação de medicamentos; Satisfação do paciente; Estudo de validação.

\begin{abstract}
The assessment of patient satisfaction with health services, using validated instruments, allows identifying the need for improvements and knowing the impact of implemented interventions. The aim of this study was to carry out the cross-cultural adaptation to the Brazilian context of a patient satisfaction questionnaire with a comprehensive pharmaceutical counseling service at hospital discharge. The questionnaire consists of nine items that assess satisfaction with the service, in addition to the patient's interest and understanding with the guidelines received at discharge. The process involved the translation, back-translation and adaptation of the items and response scales. The evaluation of the semantic, conceptual, cultural and idiomatic equivalences between the original and adapted questionnaire was carried out by a committee of experts, the degree of agreement between them being measured by the Content Validity Coefficient (CVC). The final version of the questionnaire was applied, by self-administration, to
\end{abstract}


a sample of the target population composed of 21 individuals, at the time of hospital discharge. Although the adapted instrument has presented satisfactory equivalence with the original $(\mathrm{CVC}>0.70)$, the target population had considerable difficulties in understanding it, suggesting the need to improve the items, through theoretical procedures, so that the questionnaire can be subjected to evaluation of its psychometric properties and thus be used in Brazil.

Keywords: Medication reconciliation; Patient safety; Validation study.

\section{Resumen}

La evaluación de la satisfacción del paciente con los servicios de salud, a través de instrumentos validados, permite identificar la necesidad de mejoras y conocer el impacto de las intervenciones implementadas. El objetivo de este estudio fue realizar la adaptación transcultural al contexto brasileño de un cuestionario de satisfacción del paciente con un servicio integral de entrevista farmacéutica al alta hospitalaria. El cuestionario consta de nueve preguntas que evalúan la satisfacción con el servicio, además del interés del paciente y la comprensión de las instrucciones recibidas al alta. El proceso implicó la traducción, retro traducción y adaptación del cuestionario y escalas de respuesta. La evaluación de las equivalencias semánticas, conceptuales, culturales e idiomáticas entre el cuestionario original y el adaptado fue realizada por un comité de expertos, y el grado de concordancia entre ellos se midió mediante el Coeficiente de Validez de Contenido (CVC). La versión final del cuestionario fue autoadministrada a una muestra de la población objetivo con 21 individuos en el momento del alta hospitalaria. Aunque el instrumento adaptado mostró una equivalencia satisfactoria con el original $(\mathrm{CVC}>0,70)$, la población objetivo tuvo considerables dificultades para comprenderlo, lo que sugiere la necesidad de mejorar las preguntas, a través de procedimientos teóricos, para que el cuestionario pueda ser sometido a evaluación de sus propiedades psicométricas y, así, ser utilizado en Brasil.

Palabras clave: Conciliación de Medicamentos; Seguridad del paciente; Estudio de validación.

\section{Introdução}

Durante as transições do cuidado, como na alta hospitalar, falhas na comunicação entre os diferentes profissionais e serviços de saúde podem favorecer a ocorrência de discrepâncias não-intencionais entre os medicamentos em uso pelo paciente e os recém-prescritos na nova interface do cuidado, podendo gerar erros de medicação com potencial de causar danos ao paciente (Fernandes et al., 2021; WHO, 2017, 2019). Após uma internação hospitalar, pacientes com condições clínicas complexas também estão sujeitos a alterações no seu diagnóstico e na sua farmacoterapia usual, o que pode tornar difícil o controle terapêutico sem adequada orientação (Eibergen et al., 2018).

Neste contexto, tem sido estimulado o desenvolvimento de estratégias que reduzam danos resultantes de erros ou práticas inseguras em sistemas de saúde fragmentados (WHO, 2017). Uma dessas estratégias é a implementação da Conciliação de Medicamentos (CM) nas transições do cuidado hospitalar (WHO, 2019). Enquanto serviço clínico conduzido pelo farmacêutico, o processo de CM envolve a identificação e resolução de discrepâncias medicamentosas que podem causar danos ao paciente (Conselho Federal de Farmácia, 2016), bem como o aconselhamento do paciente no momento da alta hospitalar, visando melhorar a sua compreensão sobre a farmacoterapia recém-prescrita (Fernandes, et al., 2020).

Pesquisas demonstram os benefícios clínicos da CM sob a coordenação do farmacêutico hospitalar (Mekonnen et al., 2016) e do aconselhamento de alta quando integrado a uma abordagem holística dos medicamentos prescritos para o paciente (Capiau et al., 2020). No entanto, se faz necessário considerar a percepção dos pacientes sobre as intervenções recebidas, para identificar áreas que necessitam de melhorias, bem como conhecer o impacto do serviço que está sendo implementado.

Medidas de satisfação do paciente com o atendimento hospitalar, incluindo a comunicação sobre os medicamentos e informações de alta, afetam as taxas de reembolso em sistemas de saúde privados (Centers for Medicare \& Medicaid Services, 2020), bem como são utilizadas para avaliar a qualidade de serviços farmacêuticos em hospitais públicos (Kabba et al., 2020). Neste contexto, foi desenvolvido um questionário para mensurar a satisfação dos pacientes com um programa abrangente de cuidado farmacêutico ofertado em hospitais espanhóis, denominado Consúltenos, em que a CM é liderada pelo farmacêutico na admissão e alta hospitalar, juntamente com aconselhamento sobre os medicamentos prescritos na alta (López et al., 2008). No Brasil, usa-se questionário validado para medir a satisfação dos pacientes com o serviço prestado em farmácias comunitárias (Fernandes et al., 2020), mas não há um instrumento voltado para avaliar o aconselhamento de alta hospitalar 
fornecido como um componente da CM.

Assim, este trabalho tem o objetivo de apresentar o resultado do processo de adaptação transcultural de um questionário de satisfação do paciente com um serviço abrangente de aconselhamento farmacêutico fornecido na alta hospitalar.

\section{Metodologia}

\subsection{Questionário}

O questionário de satisfação do paciente com aconselhamento farmacêutico implementado na alta hospitalar foi desenvolvido por López (2014) e é composto por nove itens que avaliam o interesse do paciente pela informação recebida (itens 1 e 2), a satisfação com a intervenção (itens 4, 5, 7 e 9) e o grau de compreensão com as orientações adquiridas (itens 6 e 8). O item 3 tem o propósito de obter informação sobre o tempo de espera do paciente para receber o serviço. As escalas de resposta são ordinais do tipo Likert de cinco pontos, que incluíam opções diferentes para os itens 5, 6 e 9 (López, 2014). Houve a autorização prévia do autor principal do instrumento para tradução e adaptação para o português do Brasil, por meio de correio eletrônico.

\subsection{Procedimentos de adaptação transcultural}

O processo de adaptação transcultural foi realizado seguindo os métodos propostos por Beaton et al. (2000) e Cassepp-Borges, Balbinotti e Teodoro (2010). Inicialmente, a versão original em espanhol foi traduzida por dois tradutores bilíngues independentes, fluentes na língua espanhola, tendo como língua-mãe o português do Brasil. As versões traduzidas foram comparadas pela equipe de pesquisa, sendo elaborada a versão consolidada em português. Nessa etapa, também se verificou a necessidade de aperfeiçoamento de itens, quando pertinente.

Os itens em português foram retrotraduzidos para o espanhol por dois tradutores bilíngues que tinham a língua espanhola como nativa. A partir das versões traduzidas e retraduzidas, foram analisados os pontos convergentes e divergentes das traduções e, os itens e escalas de respostas foram consolidados em uma versão preliminar do questionário em português.

Para análise de conteúdo inicial, a versão preliminar foi enviada para um comitê de juízes constituído por farmacêuticos doutores atuantes nas áreas de ensino e pesquisa em farmácia hospitalar e/ou assistência farmacêutica. Os especialistas avaliaram os itens traduzidos e originais quanto às equivalências semântica, conceitual, cultural e idiomática (Beaton et al., 2000), empregando a escala de Likert com três opções de resposta: "equivalente", "dúvida" e "não equivalente" (Nascimento et al., 2015). Foi permitido aos especialistas realizarem observações e proporem traduções alternativas para cada item do questionário.

Os especialistas também julgaram em qual categoria cada item se enquadrava melhor: interesse pela informação recebida (categoria 1), satisfação do paciente com a intervenção (categoria 2) ou grau de compreensão do paciente (categoria 3). Ademais, os especialistas avaliaram qual melhor opção entre os itens que geraram versões aperfeiçoadas.

A partir das respostas obtidas de pelo menos três juízes, foi calculado o Coeficiente de Validade de Conteúdo (CVC), a fim de avaliar a concordância sobre a equivalência de cada item (Cassepp-Borges et al., 2010). Considerou-se como aceitável o item que obtivesse CVC final>0,70 (Nascimento et al., 2015).

\subsection{Pré-teste}

Realizou-se um pré-teste com uma amostra de conveniência da população-alvo, a fim de verificar a compreensão do questionário. A aplicação se deu na forma de lápis e papel, por meio da autoadministração supervisionada. Sempre havia um pesquisador à disposição para observar e anotar quaisquer dúvidas e dificuldades dos participantes. 
O questionário foi aplicado em duas etapas a 21 indivíduos, internados na enfermaria de cardiologia de um Hospital Universitário, localizado no município de Vitória - ES. Os pacientes incluídos eram maiores de 18 anos, tiveram seus medicamentos conciliados e receberam aconselhamento de alta por um farmacêutico hospitalar. Na primeira etapa, quatorze pacientes participaram do estudo e, após análise dos resultados, foram realizadas modificações no questionário a fim de melhorar o entendimento nos itens que apresentaram dificuldade. Após as alterações, uma nova versão foi aplicada a sete pacientes.

\subsection{Aspectos éticos}

Este estudo faz parte de um projeto maior intitulado "Reconciliação medicamentosa realizada por farmacêutico clínico hospitalar: análise de desfechos clínicos, humanísticos e econômicos” e foi aprovado pelo Comitê de Ética em Pesquisa do hospital sob parecer $n^{\circ}$ 1.871.232 (CAAE: 61231916.0.0000.5071). Os pacientes que aceitaram participar da pesquisa realizaram a leitura e assinatura do Termo de Consentimento Livre e Esclarecido (TCLE).

\section{Resultados}

As traduções para o português foram homogêneas e identificou-se a necessidade de aperfeiçoamento dos itens 3 e 4, a fim de adequá-los para serem respondidos por uma escala de resposta ordinal, ao invés de uma resposta dicotômica (sim/não) (Tabelas 1 e 2). Na retrotradução, discrepâncias relacionadas ao vocabulário e normas gramaticais foram encontradas entre as versões.

Tabela 1 - Versões dos itens do questionário de satisfação com o aconselhamento farmacêutico durante o processo de adaptação transcultural.

\begin{tabular}{|c|c|c|c|c|}
\hline Item & Original & Versão traduzida/aperfeiçoada & Versão pré-teste & Versão pós-teste 1 \\
\hline 1 & $\begin{array}{c}\text { ¿Cuánto le interesa la información } \\
\text { verbal recibida? }\end{array}$ & $\begin{array}{c}\text { Quanto lhe interessa a informação } \\
\text { verbal recebida? }\end{array}$ & $\begin{array}{c}\text { Quanto lhe interessa a informação } \\
\text { verbal recebida? }\end{array}$ & $\begin{array}{c}\text { O quanto te interessa a informação } \\
\text { verbal que recebeu do } \\
\text { farmacêutico? }\end{array}$ \\
\hline 2 & $\begin{array}{c}\text { ¿Cuánto le interesa la información } \\
\text { escrita recibida? }\end{array}$ & $\begin{array}{l}\text { Quanto lhe interessa a informação } \\
\text { escrita recebida? }\end{array}$ & $\begin{array}{l}\text { Quanto lhe interessa a informação } \\
\text { escrita recebida? }\end{array}$ & $\begin{array}{c}\text { O quanto te interessa a informação } \\
\text { escrita que recebeu do } \\
\text { farmacêutico? }\end{array}$ \\
\hline 3 & $\begin{array}{l}\text { ¿Se ha retrasado su alta por la } \\
\text { intervención del farmacéutico? }\end{array}$ & $\begin{array}{l}\text { Houve atraso em sua alta devido à } \\
\text { intervenção do farmacêutico? / O } \\
\text { quanto sua alta foi atrasada por } \\
\text { causa do atendimento do } \\
\text { farmacêutico? }\end{array}$ & $\begin{array}{l}\text { Houve atraso em sua alta devido à } \\
\text { intervenção do farmacêutico? }\end{array}$ & Excluída \\
\hline 4 & $\begin{array}{c}\text { Si es así, ¿cree que ha merecido la } \\
\text { pena? }\end{array}$ & $\begin{array}{c}\text { Se for assim, acredita que tenha } \\
\text { valido a pena? / O quanto você } \\
\text { acredita valer a pena atrasar a alta } \\
\text { para receber atendimento do } \\
\text { farmacêutico? }\end{array}$ & $\begin{array}{c}\text { O quanto você acredita valer a penal } \\
\text { atrasar a alta para receber } \\
\text { atendimento do farmacêutico? }\end{array}$ & $\begin{array}{c}\text { O quanto você acredita valer a pena } \\
\text { atrasar a alta para receber } \\
\text { atendimento do farmacêutico? }\end{array}$ \\
\hline 5 & $\begin{array}{l}\text { ¿Cómo valoraría el trato que ha } \\
\text { recibido del farmacéutico? }\end{array}$ & $\begin{array}{l}\text { Como você avalia o atendimento } \\
\text { recebido do farmacêutico? }\end{array}$ & $\begin{array}{l}\text { Como você avalia o atendimento } \\
\text { recebido do farmacêutico? }\end{array}$ & $\begin{array}{c}\text { Como você avalia o atendimento } \\
\text { do farmacêutico? }\end{array}$ \\
\hline 6 & $\begin{array}{c}\text { ¿Ha comprendido toda la } \\
\text { información dada por el } \\
\text { farmacéutico? }\end{array}$ & $\begin{array}{l}\text { Você entendeu toda a informação } \\
\text { dada pelo farmacêutico? }\end{array}$ & $\begin{array}{l}\text { Você entendeu toda a informação } \\
\text { dada pelo farmacêutico? }\end{array}$ & $\begin{array}{l}\text { O quanto você entendeu da } \\
\text { informação dada pelo } \\
\text { farmacêutico? }\end{array}$ \\
\hline 7 & $\begin{array}{c}\text { ¿Ha podido preguntarle todas sus } \\
\text { dudas? }\end{array}$ & $\begin{array}{c}\text { Você pôde esclarecer todas as suas } \\
\text { dúvidas? }\end{array}$ & $\begin{array}{l}\text { Você pôde esclarecer todas as suas } \\
\text { dúvidas? }\end{array}$ & $\begin{array}{l}\text { Você conseguiu esclarecer todas as } \\
\text { suas dúvidas com o farmacêutico? }\end{array}$ \\
\hline 8 & $\begin{array}{l}\text { ¿Cree que conoce mejor la } \\
\text { medicación que debe tomar? }\end{array}$ & $\begin{array}{c}\text { Acredita que conhece melhor todos } \\
\text { os medicamentos que têm que } \\
\text { tomar? }\end{array}$ & $\begin{array}{c}\text { Acredita que conhece melhor todos } \\
\text { os medicamentos que têm que } \\
\text { tomar? }\end{array}$ & $\begin{array}{l}\text { Você acredita que conhece melhor } \\
\text { todos os medicamentos que têm } \\
\text { que tomar depois da orientação do } \\
\text { farmacêutico? }\end{array}$ \\
\hline 9 & $\begin{array}{c}\text { ¿Cuál es su grado de satisfacción } \\
\text { general con la entrevista? }\end{array}$ & $\begin{array}{l}\text { Qual é o seu grau de satisfação } \\
\text { geral com o atendimento do } \\
\text { farmacêutico? }\end{array}$ & $\begin{array}{l}\text { Qual é o seu grau de satisfação } \\
\text { geral com o atendimento do } \\
\text { farmacêutico? }\end{array}$ & $\begin{array}{l}\text { Qual é o seu grau de satisfação } \\
\text { geral com o atendimento do } \\
\text { farmacêutico? }\end{array}$ \\
\hline
\end{tabular}

Fonte: Questionário original López (2014). 
Tabela 2 - Versões das escalas de resposta do questionário de satisfação com o aconselhamento farmacêutico durante o processo de adaptação transcultural.

\begin{tabular}{|c|c|c|c|c|}
\hline Escalas & Original & $\begin{array}{c}\text { Versão traduzida/ } \\
\text { aperfeiçoada }\end{array}$ & Versão pré-teste & Versão pós-teste 1 \\
\hline $\begin{array}{l}\text { Para os itens } 1 \\
2,3,4,7 \text { e } 8\end{array}$ & $\begin{array}{r}\text { Mucho/ } \\
\text { Regular/1 }\end{array}$ & $\begin{array}{c}\text { Muito/Bastante/ } \\
\text { Regular/Pouco/Nada }\end{array}$ & $\begin{array}{r}\text { Muit } \\
\text { Regula }\end{array}$ & $\begin{array}{r}\text { Muito/H } \\
\text { Regular/P }\end{array}$ \\
\hline Para o item 5 & $\begin{array}{c}\text { Muy bueno/Bueno/ } \\
\text { Regular/Malo/Muy Malo }\end{array}$ & $\begin{array}{l}\text { Muito bom/Bom/ } \\
\text { Regular/Ruim/Muito ruim }\end{array}$ & $\begin{array}{l}\text { Muito bom/Bom/ } \\
\text { Regular/Ruim/Muito ruim }\end{array}$ & $\begin{array}{l}\text { Muito bom/Bom/ } \\
\text { Regular/Ruim/Muito ruim }\end{array}$ \\
\hline Para & $\begin{array}{l}\text { Todo/Casi todo/Algo/ } \\
\text { Casi nada/Nada }\end{array}$ & $\begin{array}{l}\text { Tudo/Quase tudo/Algo/ } \\
\text { Quase nada/Nada }\end{array}$ & $\begin{array}{c}\text { Tudo/Quase tudo/ } \\
\text { Alguma coisa/Quase } \\
\text { nada/Nada }\end{array}$ & $\begin{array}{c}\text { Tudo/Quase tudo/ } \\
\text { Alguma coisa/Quase } \\
\text { nada/Nada }\end{array}$ \\
\hline Para & $\begin{array}{c}\text { Muy alto/Alto/ } \\
\text { Regular/Bajo/Muy bajo }\end{array}$ & $\begin{array}{c}\text { Muito alto/Alto/ } \\
\text { Regular/Baixo/Muito baixo }\end{array}$ & $\begin{array}{c}\text { Muito alto/Alto/ } \\
\text { Regular/Pouco/Nada }\end{array}$ & $\begin{array}{c}\text { Muito alto/Alto/ } \\
\text { Regular/Pouco/Nada }\end{array}$ \\
\hline
\end{tabular}

Fonte: Questionário original López (2014).

$\mathrm{Na}$ avalição da equivalência semântica, conceitual, cultural e idiomática, em todos os itens houve grau de concordância adequado entre os juízes $(\mathrm{CVC}>0,70)$ (Tabela 3). Em relação aos itens aperfeiçoados, a versão do item 3 traduzida conforme a original teve preferência por dois juízes, sendo esta escolhida para compor o questionário. Já em relação ao item 4, a versão aperfeiçoada teve maior preferência e, assim, foi adicionada ao questionário.

Tabela 3 - Coeficiente de validade de conteúdo (CVC) dos itens, escalas de respostas do questionário e número de resposta de cada categoria.

\begin{tabular}{|c|c|c|c|c|c|c|c|}
\hline \multirow[b]{2}{*}{ Itens/Escalas de respostas } & \multicolumn{4}{|c|}{ CVC (equivalências) } & \multicolumn{3}{|c|}{ Número de respostas } \\
\hline & Semântica & Cultural & Idiomática & Conceitual & $\begin{array}{l}\text { Categoria } \\
\quad 1\end{array}$ & $\begin{array}{l}\text { Categoria } \\
\quad 2\end{array}$ & $\begin{array}{l}\text { Categoria } \\
\mathbf{3}\end{array}$ \\
\hline $\begin{array}{l}\text { Item 1- Quanto lhe interessa a informação verbal } \\
\text { recebida? }\end{array}$ & 0,741 & 0,852 & 0,963 & 0,963 & 3 & - & - \\
\hline $\begin{array}{l}\text { Item 2- Quanto lhe interessa a informação } \\
\text { escrita recebida? }\end{array}$ & 0,963 & 0,963 & 0,963 & 0,852 & 3 & - & - \\
\hline $\begin{array}{l}\text { Item 3- Houve atraso em sua alta devido à } \\
\text { intervenção do farmacêutico? }\end{array}$ & 0,963 & 0,963 & 0,963 & 0,963 & - & 3 & - \\
\hline $\begin{array}{l}\text { Item } 4-\text { Se for assim, acredita que tenha valido a } \\
\text { pena? }\end{array}$ & 0,963 & 0,963 & 0,963 & 0,963 & - & 3 & - \\
\hline $\begin{array}{l}\text { Item 5- Como você avalia o atendimento } \\
\text { recebido do farmacêutico? }\end{array}$ & 0,963 & 0,963 & 0,963 & 0,963 & - & 3 & - \\
\hline $\begin{array}{l}\text { Item 6- Você entendeu toda a informação dada } \\
\text { pelo farmacêutico? }\end{array}$ & 0,963 & 0,852 & 0,963 & 0,963 & - & - & 3 \\
\hline $\begin{array}{l}\text { Item 7- Você pôde esclarecer todas as suas } \\
\text { dúvidas? }\end{array}$ & 0,963 & 0,963 & 0,852 & 0,963 & - & - & 3 \\
\hline $\begin{array}{l}\text { Item 8- Acredita que conhece melhor todos os } \\
\text { medicamentos que têm que tomar? }\end{array}$ & 0,963 & 0,963 & 0,852 & 0,963 & - & 1 & 2 \\
\hline $\begin{array}{l}\text { Item 9- Qual é o seu grau de satisfação geral } \\
\text { com o atendimento do farmacêutico? }\end{array}$ & 0,852 & 0,852 & 0,852 & 0,741 & - & 3 & - \\
\hline Muito - Bastante - Regular - Pouco - Nada & 0,963 & 0,963 & 0,963 & 0,963 & - & - & - \\
\hline Muito bom - Bom - Regular - Ruim -Muito ruim & 0,963 & 0,963 & 0,963 & 0,963 & - & - & - \\
\hline Tudo - Quase tudo - Algo - Quase nada - Nada & 0,852 & 0,741 & 0,852 & 0,852 & - & - & - \\
\hline $\begin{array}{l}\text { Muito alto - Alto - Regular - Baixo - Muito } \\
\text { baixo }\end{array}$ & 0,963 & 0,963 & 0,963 & 0,963 & - & - & - \\
\hline Média & 0,920 & 0,929 & 0,929 & 0,929 & & - & \\
\hline
\end{tabular}

Categoria 1: interesse na informação recebida; Categoria 2: satisfação do paciente com a intervenção; Categoria 3: grau de compreensão do paciente. Fonte: Autores (com base nos dados da pesquisa).

Outros itens que obtiveram os menores CVC, foram os itens 1, em equivalência semântica, e o item 9 , em equivalência conceitual. A dúvida no item 1, foi em relação uso do pronome "lhe", mas como não houve o mesmo 
questionamento nos outros itens que usavam o mesmo formato, optou-se pela manutenção do pronome original. O item 9 gerou discussão em relação ao uso da expressão "atendimento farmacêutico", uma vez que, poderia gerar confusão em relação a qual(s) atendimento(s) recebidos durante a internação hospitalar, mas por se tratar de um questionário a ser aplicado após a orientação de alta, optou-se pela manutenção da expressão. A escala de resposta do item 6 recebeu sugestão de modificação, propondo a mudança da opção "algo" - pouco usada na cultura brasileira, para opção "alguma coisa", sendo essa alteração aceita (Tabela 2).

Os participantes do pré-teste tinham idade média de 57 anos, sendo a maioria do sexo masculino (62\%; n=13). Em relação a escolaridade, 42,76\% (9) tinham ensino médio completo, 38,00\% (8) tinham ensino fundamental incompleto, 9,52\% (2) tinha ensino fundamental completo e para 9,52\% (2) não houve resposta. Foram observadas dificuldades de compreensão pela maioria dos pacientes. Na primeira fase, em todos os itens, exceto no item 9, foi verificada pelo menos uma dúvida de compreensão. O item 3 foi o que apresentou maior dificuldade, sendo que a maioria dos participantes não entendeu a pergunta e gostaria de responder com as opções "sim/não".

Assim, foram feitas algumas alterações no questionário, como a modificação do pronome "lhe" para "te" e a flexão do verbo receber, de "recebido" para "que recebeu do farmacêutico" (Tabela 1). Ademais, optou-se pela exclusão do item 3. Na segunda fase, os pacientes tiveram menos dúvidas e consideraram de fácil entendimento, mas nos itens 7 e 8 , dois pacientes relataram desejar responder com "sim/não".

\section{Discussão}

Além de direcionar a avaliação de um serviço farmacêutico específico, para o qual ainda não existe instrumento desenvolvido no Brasil, a escolha desse questionário foi motivada por ter sido amplamente aplicado em hospitais espanhóis, com a possibilidade de avaliação do serviço em diferentes contextos, e ser composto por questões simples e de fácil aplicação (López et al., 2008). Entretanto, embora o processo de tradução tenha sido adequado e o instrumento traduzido tenha apresentado equivalência satisfatória com o original, a população-alvo teve dificuldades consideráveis em sua compreensão.

De forma geral, o questionário abrange as características ou aspectos importantes de um serviço abrangente de aconselhamento farmacêutico na alta hospitalar. Tanto a compreensão das informações pelo paciente, quanto o formato da orientação fornecida na alta (escrita ou verbal) são atributos importantes do serviço que devem ser avaliados, visto que, enquanto componente da CM, o aconselhamento de alta é utilizado também para melhorar a comunicação com o paciente e outros provedores do cuidado, com o farmacêutico fornecendo orientação oral e materiais educativos escritos sobre a farmacoterapia prescrita e suas alterações (Eibergen et al., 2018; Fernandes et al., 2020). Já a percepção do paciente sobre o atraso na alta pode ser um importante indicador de adequação do serviço, pois uma das barreiras na implementação da CM em ambiente hospitalar é a baixa espera dos pacientes para receberem o aconselhamento de alta (Sluisveld et al., 2012).

No entanto, alguns itens parecem não estar compreensíveis para população, como as questões 3, 4, 5, 6, 7 e 8, que propunham medir o grau de satisfação ou conhecimento utilizando uma escala de resposta de 5 pontos, mas com um formato de pergunta que induzia uma resposta dicotômica. Com o item 3 se buscou captar a satisfação do paciente com o atraso na alta, mas medir o quanto a alta foi atrasada não é, necessariamente, medir a satisfação do paciente com esse fato. Assim, o atraso na alta é mais adequadamente capturado pelo item 4, optando-se pela retirada do item 3 da versão final.

Tais resultados sugerem a necessidade de aperfeiçoamento do questionário, para ajustar distorções que podem não ter sido identificadas em sua construção. Embora seja comum o desenvolvimento de instrumentos baseado apenas na escolha intuitiva dos itens (Kabba et al., 2020; Pasquali, 2010), a teorização é especialmente importante quando se trabalha com satisfação do paciente, pois trata-se de um conceito ambíguo, com diversas teorias que podem orientar a construção do 
questionário (Batbaatar et al., 2015).

Outra característica importante do instrumento foi o uso de quatro escalas de respostas diferentes. Esses formatos são comuns na avaliação das experiências do paciente, a pesquisa Hospital Consumer Assessment of Healthcare Providers and Systems (HCAHPS), por exemplo, contém 19 itens sobre aspectos críticos do cuidado hospitalar, com diferentes escalas de respostas (Centers for Medicare \& Medicaid Services, 2020). Entretanto, é importante a observância de critérios que tornem os itens mais adequados para captarem as dimensões do construto, em acordo com a pergunta construída (Pasquali, 2010).

Em relação aos aspectos de adaptação transcultural, as questões 5 e 9 em português parecem ter focado em um mesmo atributo do serviço, o "atendimento". Originalmente o item 5 propunha avaliar o "trato" do farmacêutico, talvez procurando avaliar os aspectos subjetivos, como cordialidade, cortesia ou respeito com o que o profissional atende ao paciente, enquanto o item 9 procura resumir a satisfação com o serviço considerando todos os seus aspectos. Assim, os itens originais e os recém adaptados, carecem de uma análise semântica que verifique os aspectos de validade aparente (Pasquali, 2010), podendo-se consultar os autores do questionário original para compreender melhor os construtos a serem medidos, bem como especialistas em idiomas e representantes da população-alvo (Ferreira et al., 2014; Fortes \& Araújo, 2019).

Neste contexto, é importante ressaltar o impacto da adaptação também no formato de aplicação do questionário para o contexto brasileiro. Na pesquisa original, os pacientes respondiam o questionário no hospital ou em casa, enviando posteriormente aos pesquisadores via correio (López et al., 2008). Ao aplicar o questionário logo após a alta hospitalar, podese encontrar dificuldade na adesão dos pacientes e potencializar o viés de gratidão, enquanto se reduz o potencial viés de memória (Esperidião \& Trad, 2005).

Conforme exposto, esse estudo apresenta limitações intrínsecas ao instrumento, bem como ao processo de adaptação transcultural. Embora tenha sido seguido as etapas de tradução recomendadas internacionalmente, obtendo-se equivalências apropriadas entre as versões original e adaptada, a ausência de especialistas de idiomas na avaliação das traduções pode ter prejudicado a adequada adaptação do instrumento. Por outro lado, o pré-teste realizado em pequenos grupos, facilitou a identificação de falhas e o ajuste imediato no instrumento, conforme relatado também por outros autores (Ferreira et al., 2014).

\section{Conclusão}

As evidências de validade obtidas até aqui para o questionário adaptado demonstram que além da avaliação de suas propriedades psicométricas é preciso um longo trabalho de aperfeiçoamento dos itens para que possam medir de forma consistente e confiável a satisfação dos pacientes com um serviço abrangente de aconselhamento farmacêutico fornecido na alta hospitalar.

\section{Agradecimentos}

Este estudo foi parcialmente financiado pela Fundação de Amparo à Pesquisa e Inovação do Espírito Santo (FAPES); Conselho Nacional de Desenvolvimento Tecnológico Científico e Tecnológico (CNPq); Departamento de Ciência e Tecnologia (Decit) da Secretaria de Ciência, Tecnologia, Inovação e Insumos Estratégicos em Saúde, (SCTIE) do Ministério da Saúde (MS); Secretaria Estadual de Saúde do Espírito Santo (SESA) através do Programa de Pesquisa para o Sistema Único de Saúde: Gestão da Saúde Compartilhada (Edital n³/2018 - PPSUS), e também pela Coordenação de Aperfeiçoamento de Pessoal de Nível Superior (CAPES). As agências de financiamento acima não desempenharam nenhum papel direto nem no desenho do estudo, nem na coleta, análise e interpretação dos dados, nem na redação do artigo, nem na decisão de submetê-lo para publicação. 


\section{Referências}

Batbaatar, E., Dorjdagva, J., Luvsannyam, A., \& Amenta, P. (2015). Conceptualisation of patient satisfaction: A systematic narrative literature review. Perspectives in Public Health, 135(5), 243-250. 10.1177/1757913915594196

Beaton, D. E., Bombardier, C., Guillemin, F., \& Ferraz, M. B. (2000). Guidelines for the process of cross-cultural adaptation of self-report measures. Spine, 25(24), 3186-3191. 10.1097/00007632-200012150-00014

Capiau, A., Foubert, K., Van der Linden, L., Walgraeve, K., Hias, J., Spinewine, A., Sennesael, A. L., Petrovic, M., \& Somers, A. (2020). Medication Counselling in Older Patients Prior to Hospital Discharge: A Systematic Review. Drugs and Aging, 37(9), 635-655. 10.1007/s40266-020-00780-

Cassepp-Borges, V., Balbinotti, M., \& Teodoro, M. (2010). Tradução e Validação de conteúdo: Uma proposta para adaptação de instrumentos. In Instrumentação Psicológica: Fundamentos e Práticas (pp. 506-520). Artmed.

Centers for Medicare \& Medicaid Services. (2020). HCAHPS: Patients' Perspectives of Care Survey. https://www.cms.gov/Medicare/Quality-InitiativesPatient-Assessment-Instruments/HospitalQualityInits/HospitalHCAHPS

Conselho Federal de Farmácia. (2016). Serviços farmacêuticos diretamente destinados ao paciente, à família e à comunidade: contextualização e arcabouço conceitual. Brasília: Conselho Federal de Farmácia.

Eibergen, L., Janssen, M. J. A., Blom, L., Karapinar-Çarkit, F. (2018). Informational needs and recall of in-hospital medication changes of recently discharged patients. Research in Social and Administrative Pharmacy, 14(2), 146-152. 10.1016/j.sapharm.2017.01.006</div>

Esperidião, M., \& Trad, L. A. B. (2005). Avaliação de satisfação de usuários. Ciência \& Saúde Coletiva, 10, 303-312.

Fernandes, B. D., Almeida, P. H. R. F., Foppa, A. A., Sousa, C. T., Ayres, L. R., \& Chemello, C. (2020). Pharmacist-led medication reconciliation at patient discharge: A scoping review. Research in Social and Administrative Pharmacy, 16(5), 605-613. /10.1016/j.sapharm.2019.08.001

Fernandes, B. D., Freitas, R. R., Melchiors, A. C., \& Pontarolo, R. (2020). Satisfação dos usuários com serviços da farmácia comunitária: uma abordagem espacial. Revista de Atenção à Saúde, 17(62), 12-23. 10.13037/ras.vol17n62.6107

Fernandes, B. D., Ribeiro, L.C., Santos, J. C. P., Ayres, L. R., Chemello, C. (2021). Medication Reconciliation at hospital admission and discharge: Evaluation of fidelity and process outcomes in a real-world setting. International Journal of Clinical Practice, 75(10):e14656. 10.1111/ijcp.14656

Ferreira, L., Neves, A. N., Campana, M. B., \& Tavares, M. C. G. C. F. (2014). Guia da AAOS/IWH: sugestões para adaptação transcultural de escalas. Avaliação Psicológica, 13(3), 457-461.

Fortes, C. P. D. D., \& Araújo, A. P. Q. C. (2019). Check list para tradução e Adaptação Transcultural de questionários em saúde. Cadernos Saúde Coletiva, 27(2), 202-209. 10.1590/1414-462x201900020002

Kabba, J. A., Bah, A. J., James, P. B., Chang, J., Kitchen, C., Jiang, M., Zhao, M., \& Fang, Y. (2021). Patients satisfaction with free healthcare pharmaceutical services in Sierra Leone: a national cross-sectional study. International Journal of Clinical Pharmacy, 43, 556-565. 10.1007/s11096-020-01163-5

López, M. Á. P. (2014). Programa consúltenos de información al alta hospitalaria: diseño, puesta en marcha y evaluación de su impacto clínico y humanístico. Universidad Miguel Hernández de Elche.

López, M. Á. P., Saliente, M. T. A., \& Company, E. S. (2008). CONSULTENOS: Programa de información al alta hospitalaria. Desarrollo y resultados del primer año de funcionamiento en 5 hospitales. Farmacia Hospitalaria, 32(6), 323-330. 10.1016/S1130-6343(08)76280-9

Mekonnen, A. B., McLachlan, A. J., \& Brien, J. E. (2016). Effectiveness of pharmacist-led medication reconciliation programmes on clinical outcomes at hospital transitions: a systematic review and meta-analysis. BMJ Open, 6(2), e010003. 10.1136/bmjopen-2015-010003

Nascimento, E., Coutinho, A. C. A. M., Andrade, J. E., \& Mendonça, L. M. M. (2015). Adaptação transcultural da Workplace Deviance Scale (WDS) para o contexto brasileiro. Revista Psicologia Organizações e Trabalho, 15(4), 384-396. 10.17652/rpot/2015.4.649

Pasquali, L. (2010). Testes referentes a constuto: teoria e modelo de construção. In Instrumentação Psicológica: Fundamentos e Práticas (p. 560). Artmed.

Sluisveld, N., Zegers, M., Natsch, S., \& Wollersheim, H. (2012). Medication reconciliation at hospital admission and discharge: Insufficient knowledge, unclear task reallocation and lack of collaboration as major barriers to medication safety. BMC Health Services Research, 12(170), 1-12. 10.1186/1472-6963$12-170$

WHO. (2017). Medication Without Harm - Global Patient Safety Challenge on Medication Safety. World Health Organization.

WHO. (2019). Medication Safety in Transitions of Care. World Health Organization. 